\title{
Differential patterns of antimicrobial resistance in population subsets of Escherichia coli isolated from waste- and surface waters
}

\author{
Vânia Figueira, Elizabete Serra, Célia M. Manaia* \\ CBQF/Escola Superior de Biotecnologia, Universidade Católica Portuguesa, 4200-072 Porto, Portugal
}

Keywords:

Escherichia coli

Antimicrobial resistance

Integrons

Phylogenetic groups

Water

\begin{abstract}
The species Escherichia coli comprises different subgroups with distinct phylogeny, physiology and ecology and, thus, presumably, with different roles in antimicrobial resistance dissemination. E. coli strains isolated from raw and treated municipal wastewater and from urban water streams were characterized in terms of phylogenetic groups, antimicrobial resistance patterns and the presence of class 1 and class 2 integrons. Our main objective was to investigate the contribution of the different phylo-groups in antimicrobial resistance dissemination in urban waters. Groups A and B1 were predominant in all types of water, evidencing, respectively, the lowest and the highest resistance prevalence. Municipal wastewater treatment was accompanied by significant increases of ciprofloxacin and streptomycin resistance $(\mathrm{p}<0.01)$. Antimicrobial resistance prevalence differed significantly between the different phylo-groups and within the same group, mainly in group A. Such differences contributed to explain the higher ciprofloxacin and streptomycin resistance rates observed in treated effluent in comparison with the raw wastewater. We conclude that the dynamics of the bacterial populations has a major role on the dissemination of antimicrobial resistance in the environment.
\end{abstract}

\section{Introduction}

Given the close contact that bacteria of the species Escherichia coli have with humans and other warm blooded animals, as well as their recognized ubiquity, these organisms are amongst the most important vectors in the dissemination of antimicrobial resistance in the environment. The species $E$. coli comprehends different phylogenetic lineages, often with distinct patterns of distribution among human populations, animals or the environment (Herzer et al., 1990; Walk et al., 2007; Gordon et al., 2008). These phylogenetic lineages have been clustered into four groups, named A, B1, B2 and D, for which Clermont et al. (2000) developed a rapid and inexpensive PCR-based diagnostic method. Numerous studies have used the Clermont et al. (2000) PCRbased screening method, allowing the comparison between studies with isolates from different regions and origins (e.g. Maynard et al., 2004; Walk et al., 2007; Sabaté et al., 2008; Skurnik et al., 2008; Cortés et al., 2010).

According to previous descriptions, those four phylogenetic groups of $E$. coli may differ in terms of ecology and physiology. For instance, groups A and B1 have been referred to as more prevalent among human populations, whereas groups B2 and D are described as comprising significantly more pathogenic strains (Duriez et al. 2001;

* Corresponding author. Escola Superior de Biotecnologia, Universidade Católica Portuguesa, 4200-072 Porto, Portugal. Tel.: + 35122 5580059; fax: + 351225090351. E-mail address: cmmanaia@esb.ucp.pt (C.M. Manaia).
Maynard et al., 2004; Hamelin et al., 2007; Skurnik et al., 2008). Additionally, the influence of some external factors on the dynamics of the different $E$. coli phylogenetic groups was emphasized in different studies (Duriez et al., 2001; Orsi et al., 2007; Skurnik et al., 2008). These studies led to the hypothesis that in the external environment, namely in waters, $E$. coli populations may suffer different rearrangements. For instance, in a study with beach freshwater isolates, Walk et al. (2007) observed the predominance of members of the group B1, concluding about the persistence and adaptive advantage of these organisms outside the host. The analyses of the distribution of antimicrobial resistance amongst the different $E$. coli phylogenetic groups have been addressed in some studies. In general, these studies reported a trend for a lower antimicrobial resistance prevalence in groups B2 and/or D (Skurnik et al., 2005; Rijavec et al., 2006; Garcia-Aljaro et al., 2009; Mataseje et al., 2009).

Municipal wastewater and surface waters, being receptors of gut microbiota of humans and other animals, constitute important vehicles in the dissemination of $E$. coli in the urban environment. Thus, studies on the distribution of phylogenetic groups in this type of environment and its association with antimicrobial resistance and integron patterns will contribute to assess the modes of resistance dissemination. In spite of this, to our knowledge, information on E. coli phylogenetic groups in municipal wastewater and in urban surface waters and its relationship with antimicrobial resistance and class 1 and class 2 integrons is scant. In the same way, studies aiming at establishing a relationship between antimicrobial resistance and 
phylogenetic grouping in environmental E. coli are rare. In the present work we investigated the diversity of cultivable E. coli in waste- and surface waters and its relationship with antimicrobial resistance and integron prevalence. Specifically, we aimed at addressing the following aspects: i) which are the major phylogenetic groups in municipal waste- and urban surface waters; ii) if municipal wastewater treatment implies significant rearrangements in the proportions of each phylogenetic group; and iii) which, if any, of the phylogenetic groups, or of the respective genotypes, presents higher prevalence of antimicrobial resistance and of class 1 and class 2 integrons.

\section{Materials and methods}

\subsection{Water samples and bacterial isolates}

E. coli strains analysed in this study were isolated from wastewater (8 sampling campaigns) and surface water (5 sampling campaigns) collected from January 2004 to November 2006 in the region of Porto in northern Portugal. Wastewater samples included RWW and TWW of a municipal wastewater treatment facility (Ferreira da Silva et al., 2007). Surface water samples were collected from urban WS, where inadvertent human and/or animal sewage discharge by houses not connected to the municipal sewage collector was supposed to occur. $E$. coli strains analyzed in this study were isolated on different culture media. Wastewater strains (22 isolates) were isolated on Plate Count Agar (Pronadisa), on m-endo-agar-LES (Difco) (64 isolates) and on mFC agar (Difco) (257 isolates, identified previously as described by Ferreira da Silva et al., 2007). WS strains were isolated during the routine monitoring of microbiological water quality and, thus, water samples were processed according to the international standard methods (International Organization for Standardization, ISO 93081:2000), with the isolates being recovered on the medium Lactose TTC (Oxoid). After purification, presumable E. coli were cryopreserved at $-80{ }^{\circ} \mathrm{C}$ in nutrient broth with $15 \%(\mathrm{v} / \mathrm{v})$ of glycerol for further analysis. The bacteria recovered on Plate Count Agar, m-endo-LES agar and Lactose TTC agar were identified as described before for the m-FC agar isolates (Ferreira da Silva et al., 2007). After removing possible repetitions (isolates recovered in the same sampling campaign, on the same culture medium plate, with the same RAPD profile, with a similar antimicrobial resistance pattern, with the same Clermont et al. (2000) genotype and, when applicable, with identical integron profile) was settled a collection of $460 \mathrm{E}$. coli isolates.

\subsection{PCR screening of phylogenetic groups}

E. coli phylogenetic groups were screened using the method of Clermont et al. (2000). Isolates were classified according to the combination of the PCR amplification of the genes chuA and yjaA and the DNA fragment TSPE4-C2, with the genotypes $-/-/-\left(=\mathrm{A}_{0}\right)$ and $-/$ $+/-\left(=A_{1}\right)$ corresponding to group $A,-/-/+$ to group $B 1,+/+/-$ $\left(=\mathrm{B} 2_{1}\right)$ and $+/+/+\left(=\mathrm{B} 2_{2}\right)$ to group $\mathrm{B} 2$, and $+/-/-\left(=\mathrm{D}_{1}\right)$ and $+/$ $-/+\left(=D_{2}\right)$ to group $\mathrm{D}$. In preliminary assays, the authenticity of each amplicon was confirmed by sequencing analysis. Positive reactions, confirmed by sequencing were included in each PCR assay as positive controls. Additionally, about $10 \%$ of the PCR products, selected randomly, were sequenced for confirmation of the authenticity. In isolates with the genotype $A_{0}$, besides the analysis of the $16 \mathrm{~S}$ rRNA gene sequence, also the presence of the gene lac $Z$ was screened, as recommended by Higgins et al. (2007).

\subsection{Determination of antimicrobial resistance phenotypes}

Antimicrobial resistance phenotypes were determined for 12 antimicrobial compounds (AML, $25 \mu \mathrm{g}$; TIC, $75 \mu \mathrm{g}$; CP, $30 \mu \mathrm{g}$; CAZ, $30 \mu \mathrm{g}$; MEM, $10 \mu \mathrm{g}$; CT, $50 \mu \mathrm{g}$; SUL, $25 \mu \mathrm{g}$; SXT, $25 \mu \mathrm{g}$; CIP, $5 \mu \mathrm{g}$; TET,
$30 \mu \mathrm{g}$; GEN, $10 \mu \mathrm{g}$; STR, $10 \mu \mathrm{g}$ ) using the agar diffusion method, as described previously (Ferreira da Silva et al., 2006; CLSI, 2007). For AML, CT and SUL, not included in CLSI standards for Enterobacteriaceae, the criteria were $S \geq 21$ and $R<14, S \geq 10$ and $R<10$ and $S \geq 16$ and $R<12$, respectively. Whenever, diameters larger than $R$ but smaller than $S$ were observed, were referred to as intermediary, and these were excluded from calculations. The strains E. coli ATCC 25922 and Pseudomonas aeruginosa DSM 1117 (=ATCC 27853) were included in each experimental set as quality and reproducibility controls.

\subsection{Detection of class 1 and 2 integrons}

All the isolates under study were screened for the presence of class 1 and class 2 integrons. Class 1 integrons were screened using two methods, the detection of the variable region contained within the conserved sequence CS3-CS5, as described before (Lévesque et al., 1995; Ferreira da Silva et al., 2007) and the detection of the integrase gene (Henriques et al., 2006). The content of the variable region was determined through sequencing analysis with the primer CS5 and/or mapped using the primers aadA1 rv (ant(3")-la, Lévesque et al., 1995), aadA1 fw (ant(3")-I-3', Lévesque et al., 1995) and dhfrl (Lévesque et al., 1995; Ferreira da Silva et al., 2007). For sequencing analysis, PCR products of the variable region of class 1 integrons were cloned into a pGEM®-T easy vector (pGEM ${ }^{\circledR}-\mathrm{T}$ Easy Vector System, Promega). DNA from clones was extracted, amplified and sequenced with universal primers M13.

Class 2 integrons were screened with the primer pairs aadA1 fw/ TnsE-Tn7 (5'-GAA TTC GAC ATG TTT GGA CGC CTT GGC-3') and aadA1 rv/IntI2 fw (5'-TTA TTG CTG GGA TTA GGC-3') (Laroche et al., 2009), in $10 \mu \mathrm{L}$ of reaction mixture ( $0.4 \mathrm{U}$ Taq Polymerase (Fermentas), $1.5 \mathrm{mM}$ $\mathrm{MgCl}_{2}, 1 \times$ Taq buffer, $200 \mu \mathrm{M}$ dNTP mixture, $0.4 \mu \mathrm{M}$ of each primer and $1 \mu \mathrm{L}$ of crude cell lysate). The thermalcycler (Biometra) programming consisted in a first step of 5 min at $94^{\circ} \mathrm{C}$, followed by 30 cycles of $30 \mathrm{~s}$ at $94{ }^{\circ} \mathrm{C}, 30 \mathrm{~s}$ at $55 / 48{ }^{\circ} \mathrm{C}$ (respectively) and $6 \mathrm{~min}$ at $72{ }^{\circ} \mathrm{C}$, and a final extension step of $7 \mathrm{~min}$ at $72^{\circ} \mathrm{C}$ (Laroche et al., 2009). The class 2 integron content was assessed by sequencing analysis. Positive controls, previously established on basis of sequencing analysis were included in each PCR experimental set.

\subsection{Screening of genetic determinants for ciprofloxacin resistance}

Ciprofloxacin resistant isolates $(n=23)$ were screened for the presence of mutations in the genes gyrA and parC. The nucleotide sequences of the amplicons generated, respectively, with the primers gyrA6/gyrA631R and HJL3/HJL4 were analysed for the presence of missense substitution mutations, as described before (Weigel et al., 1998; McDonald et al., 2001). The presence of the genes qnrA, qnrB and qnrS was screened in isolates yielding a ciprofloxacin resistant or intermediary $(\mathrm{n}=30)$ phenotype, using the multiplex PCR method described previously (Cattoir et al., 2007). The positive controls, strains E. coli Lo (qnrA1+), Klebsiella pneumoniae B1 (qnrB1+) and Enterobacter cloacae S1 (qnrB4+ and qnrS1+), were included in each PCR assay.

\subsection{Statistical analyses}

The percentages of the different phylogenetic groups and of the corresponding genotypes, of antimicrobial resistance and of integrons were compared using the chi-square test (SPSS 17.0 for Windows). A significance value of $\mathrm{p}<0.05$ was considered.

\section{Results}

\subsection{Distribution of phylogenetic groups}

The set of $460 \mathrm{E}$. coli isolates examined in this study included 204 from RWW, 139 from TWW and 117 from WS. Among the four 
phylogenetic groups, group A was the most prevalent in all types of water (47.5\% in RWW, $41.7 \%$ in TWW and $43.6 \%$ in WS), followed by group B1 (24.0\% in RWW, 21.6\% in TWW and 21.4\% in WS) and by groups B2 in wastewater (18.6\% in RWW and $22.3 \%$ in TWW) and D in water streams (24.8\%). The least prevalent groups were D in wastewater (9.8\% in RWW and $14.4 \%$ in TWW) and B2 in water streams (10.3\%). The prevalence of group B2 was significantly lower in water streams than in wastewater $(p<0.001)$, while the opposite was observed for group $\mathrm{D}$, which prevalence was higher in water streams $(p<0.005)$ (Fig. 1a). The distribution of both genotypes comprising each phylogenetic group $\left(A_{0} / A_{1}, B 2_{1} / B 2_{2}, D_{1} / D_{2}\right)$ was slightly but not significantly different, except for group $\mathrm{B} 2$, in which was observed an evident disproportion between both genotypes, due to the rarity of the type $\mathrm{B} 2{ }_{1}$ (Fig. 1b).

\subsection{Antimicrobial resistance vs. phylogenetic group}

Among the antimicrobial substances tested, sulfamethoxazole resistance prevalence was the highest (77.6\%), followed by tetracycline (26.3\%), streptomycin (23.5\%) and the $\beta$-lactam antibiotics (amoxicillin 23.7\%, ticarcillin $22.8 \%$ and cephalothin 18.0\%). The analysis of antimicrobial resistance prevalence over the four phylogenetic groups (Fig. 2a) showed that, in general, group A presented lower rates of antimicrobial resistance than the other groups. No resistance was found against meropenem and ceftazidime, and only one strain, of the type $A_{0}$ and isolated from RWW, yielded resistance to colistin. Gentamicin resistance was also rare, observed in 17 isolates of the different phylogenetic groups $\left(2 \mathrm{~A}_{0} ; 3 \mathrm{~A}_{1} ; 6 \mathrm{~B} 1 ; 3 \mathrm{~B} 2_{2}\right.$ and $3 \mathrm{D}_{1}$ ).

Comparing the resistance prevalence between the genotypes that constitute each phylogenetic group (Fig. 2b) it was observed that the most relevant differences occurred in group $A$. In comparison with the strains of the type $A_{1}$, isolates of the type $A_{0}$ yielded significantly lower prevalence of resistance to five out of eight antimicrobial compounds tested. Additionally, in group B2, both types differed significantly in the resistance prevalence to ticarcillin and streptomycin and in group $\mathrm{D}$,

a
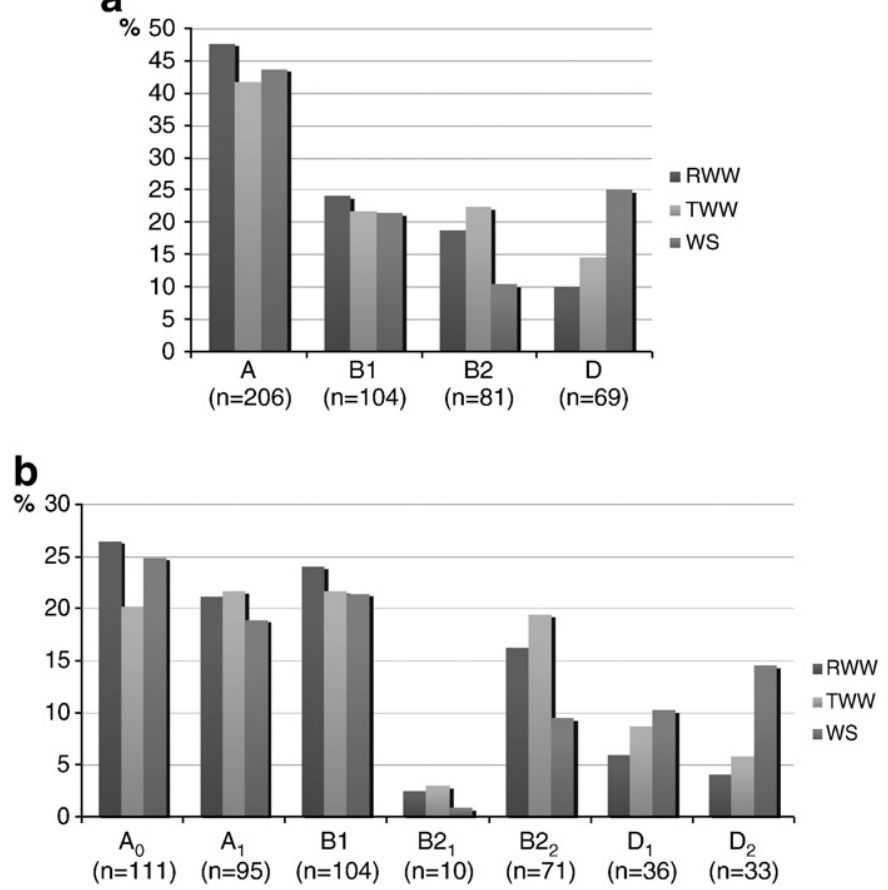

Fig. 1. Prevalence of $E$. coli in raw wastewater (RWW), treated wastewater (TWW) and water streams (WS), (a) according to the respective phylogenetic groups (A, B1, B2, D) and (b) the corresponding genotypes. both types differed significantly in resistance prevalence to cephalothin, ciprofloxacin and streptomycin.

Resistance to three or more (R3) or five or more (R5) antimicrobial compounds was observed to be significantly less prevalent in group A than in the others $(R 3, p<0.05 ; R 5, p \leq 0.005)$ (Table 1$)$. Such a result was explained by the differences observed between the two genotypes comprising this phylogenetic group, as among the isolates with the genotype $A_{0} R 3$ and R5 were significantly less prevalent $(p<0.05)$ than in those of the type $A_{1}$. For group B2 such a comparison is biased due to the low number of isolates with the genotype $\mathrm{B} 2_{1}$ and in group D it was observed that the relationship between the percentage of R3 or of R5 and the genotype were dependent on the type of water (Table 1 ).

\subsection{Class 1 and class 2 integrons vs. phylogenetic groups}

A total of 90 isolates, out of the 460 under study (19.6\%), had a class1 integron, detected through the integrase gene and/or of the conserved regions CS3/CS5 (RWW 19.1\%, TWW 22.3\% and WS 17.1\%), and $23(5.0 \%$ ) had a class 2 integron (RWW 4.9\%, TWW 5.8\% and WS $4.3 \%$ ). Of the 90 isolates with a class 1 integron, in 87 (18.9\% of the 460 isolates) was detected the intI 1 gene (RWW 18.1\%, TWW 21.6\% and WS $17.1 \%$ ), whereas in only 36 ( $7.8 \%$ of the 460 isolates) was detected the CS3/CS5 region (RWW 7.8\%, TWW 12.2\% and WS 2.6\%). In three of the 36 isolates in which the regions CS3/CS5 were successfully targeted, was not observed the integrase gene (3.3\% of the isolates carrying class 1 integron).

The patterns of antimicrobial resistance observed in isolates carrying a class 1 or a class 2 integron were similar and included resistance to sulfamethoxazole (88.9\% and $91.3 \%$, respectively) and streptomycin (64.4\% and $95.7 \%$ ), tetracycline (64.4\% and $60.9 \%)$, the $\beta$-lactams amoxicillin (61.1\% and $60.9 \%$ ) and ticarcillin $(61.1 \%$ and $56.5 \%$ ) and sulfamethoxazole/trimethoprim (60.0\% and $52.2 \%$ ). In class 1 integrons, for which the variable region was investigated, i.e., those for which the regions flanked by the conserved segments CS3' and CS5' were successfully amplified, the genes more frequently found were aadA1 and/or dfrA1. The cassette aadA5/dfrA17 was observed in five isolates (four belonging to group $B 2$ and one to group $D$ ), the cassette aadA2/dfrA12 was present in a single isolate (from WS, belonging to group A) and the gene aadA2 was also observed in a single isolate (from TWW, belonging to group D). Class 2 integrons were observed to contain one of three types of gene cassette: sat2/aadA1, dfrA1/sat2/ aadA1 and dfrA1/estX/sat2/aadA1. The simultaneous presence of a class 1 and a class 2 integron was observed in two isolates from TWW belonging to the groups B1 and B2.

In general, class 1 integrons were significantly less prevalent in group $A(p<0.001)$ than in the others. The inability to target the conserved regions CS3/CS5 was observed in every group, being more notorious in approximately two thirds of the isolates of the groups A and B1 (Table 1). The distribution of class 1 integrons over the different genotypes comprising each phylogenetic group followed a pattern coherent with that observed for R3 and R5, with the type $A_{0}$ presenting a significantly lower prevalence $(p<0.01)$ than the other types. Isolates of the type $A_{0}$ presented also the lowest prevalence of class 2 integrons. In contrast, types $A_{1}$ and $D_{2}$ presented the heaviest distribution of class 2 integrons (Table 1 ). In WS, class 2 integrons were observed exclusively in isolates of these types.

\subsection{Effects of wastewater treatment}

Wastewater treatment had no effect on the distribution of the phylogenetic groups, as no significant differences were observed between raw and treated wastewater for the different groups. However, within the phylogenetic group $A$, the type $A_{0}$ was significantly more prevalent in RWW (26.5\%) than in TWW $(20.1 \%$, $\mathrm{p}<0.05$ ). 
a

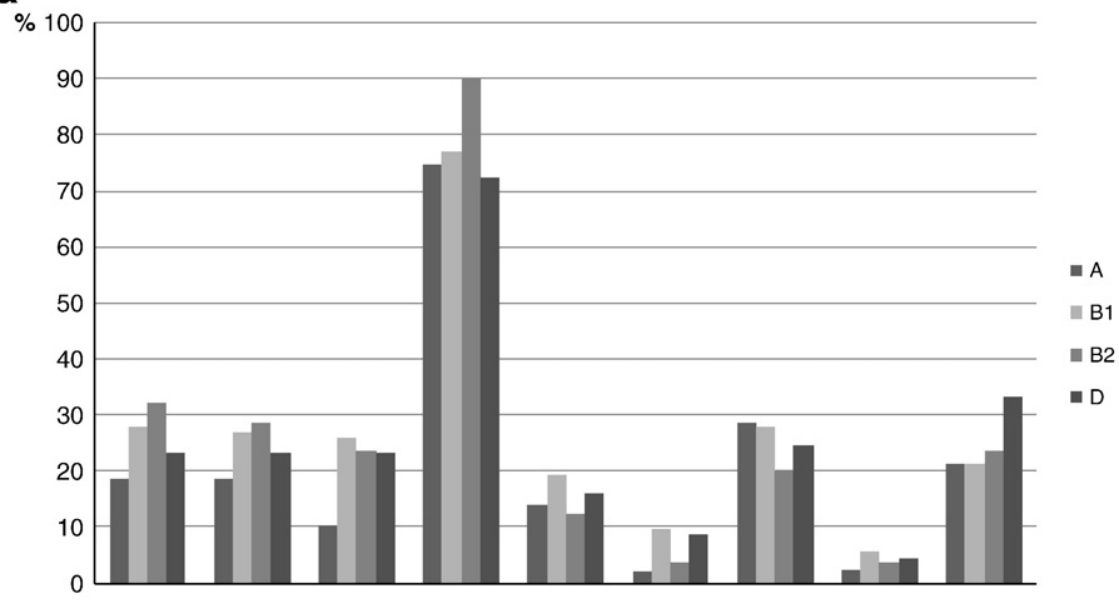

\begin{tabular}{|c|c|c|c|c|c|c|c|c|c|c|}
\hline & & AML & TIC & $\mathrm{CP}$ & SUL & SXT & CIP & TET & GEN & STR \\
\hline \multirow{3}{*}{ A } & B1 & $\mathrm{B} 1>\mathrm{A}$ & $\mathrm{B} 1>\mathrm{A}$ & $B 1>A$ & & & $B 1>A$ & & & \\
\hline & B2 & $B 2>A$ & $B 2>A$ & $B 2>A$ & $\mathrm{~B} 2>\mathrm{A}$ & $A>B 2$ & & $A>B 2$ & & \\
\hline & D & & & $D>A$ & & & $D>A$ & & & $D>A$ \\
\hline \multirow{2}{*}{ B1 } & B2 & & & & $\mathrm{B} 2>\mathrm{B} 1$ & $\mathrm{~B} 1>\mathrm{B} 2$ & $\mathrm{~B} 1>\mathrm{B} 2$ & $\mathrm{~B} 1>\mathrm{B} 2$ & & \\
\hline & D & & & & & & & & & $\mathrm{D}>\mathrm{B} 1$ \\
\hline B2 & D & & & & $B 2>D$ & & & & & \\
\hline
\end{tabular}

b

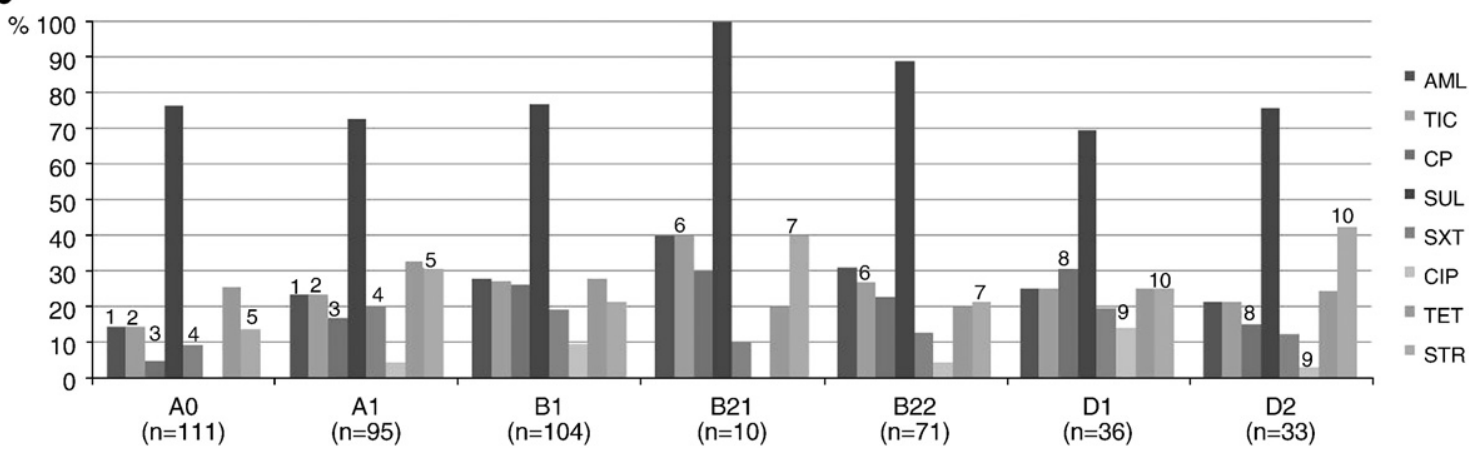

Fig. 2. Prevalence (\%) of antimicrobial resistance in each (a) of the four phylogenetic groups and indication of the significantly different prevalence values ( $<<0.05$ ); and (b) genotype and comparison of the prevalence values between both types of the same phylogenetic group (numbers above the bar indicate the significant differences observed, $\mathrm{p}<0.05$ ).

The prevalence of $\mathrm{R} 5$, but not of $\mathrm{R} 3$, was observed to be significantly higher in TWW $(24 \%)$ than in RWW $(17 \%, \mathrm{p}<0.05)$ (data not shown). The most frequent resistance phenotypes in the 71 wastewater isolates with a R5 phenotype were: sulfamethoxazole (97.2\%), amoxicillin (94.4\%), ticarcillin (91.5\%), streptomycin (84.5\%), tetracycline (76.0\%), sulfamethoxazole/trimethoprim (64.8\%), cephalothin (40.8\%), ciprofloxacin (28.2\%) and gentamicin (15.5\%). A total of 48 of the R5 wastewater isolates (68\%) were members of the groups A or B1, and only 9 (13\%) belonged to group D.

It was not possible to attribute the higher percentage of R5 observed in TWW than in RWW to a single phylogenetic group. However, among the isolates of the type $A_{1}$, R5 was significantly $(p \leq 0.05)$ more prevalent in TWW than in RWW (Table 1). Wastewater treatment was accompanied by variations on the prevalence of class 1 integrons, although such an effect did not follow a similar trend in all genotypes. A reduction was observed for the types $A_{0}, B 2_{2}$ and $D_{1}$, whereas the opposite was observed for the types $A_{1}, B 1$ and $D_{2}$. Although these variations were not significant, this pattern coincided with that observed for R3 and R5 prevalence (Table 1).
Wastewater treatment was accompanied by significant variations on antimicrobial resistance prevalence of ciprofloxacin and streptomycin. In these cases, resistance prevalence values were significantly higher in TWW than in RWW (8.6\% vs.3.4\%, $<<0.01$ for ciprofloxacin, and $31.7 \%$ vs. $22.5 \%, \mathrm{p} \leq 0.005$ for streptomycin). Wastewater isolates resistant to ciprofloxacin and streptomycin were also resistant to sulfamethoxazole (91.3\% and $91.7 \%$ of the times, respectively), to tetracycline ( $91.3 \%$ and $66.7 \%$ ), to amoxicillin (87.0\% and $67.6 \%$ ) and ticarcillin (87.0\% and $66.7 \%$ ), to sulfamethoxazole/trimethoprim (60.9\% and $52.8 \%$ ), to cephalothin (43.5\% and 28.7\%) and to gentamicin (21.7\% and 9.3\%). More than $70 \%$ of the ciprofloxacin resistant isolates were also resistant to streptomycin. A class 1 integron was detected in $69.6 \%$ of the isolates with resistance to ciprofloxacin and in $53.7 \%$ of those with resistance to streptomycin. Class 2 integrons were detected in $17.4 \%$ and $20.4 \%$ of the ciprofloxacin and streptomycin resistant isolates, respectively.

Mutations in the topoisomerase genes gyrA and parC were associated with the ciprofloxacin resistance phenotypes observed of. In the gene gyrA two point mutations, in the positions 83 and 87 were observed. Twenty two out of the 23 isolates presented a serine to 
Table 1

Prevalence (\%) of resistance to three or more (R3) and to five or more (R5) antibiotics, and prevalence (\%) of integrons observed within each phylogenetic group and genotype in raw wastewater (RWW), treated wastewater (TWW) and water streams (WS).

\begin{tabular}{|c|c|c|c|c|c|c|c|c|}
\hline & \multirow[t]{2}{*}{ Origin } & \multicolumn{2}{|l|}{ A } & \multirow{2}{*}{$\frac{B 1}{(n=104)}$} & \multicolumn{2}{|l|}{ B2 } & \multicolumn{2}{|l|}{$\mathrm{D}$} \\
\hline & & $A_{0}(n=111)$ & $A_{1}(n=95)$ & & $\mathrm{B} 2_{1}(\mathrm{n}=10)$ & $\mathrm{B} 2_{2}(\mathrm{n}=71)$ & $D_{1}(n=36)$ & $\mathrm{D}_{2}(\mathrm{n}=33)$ \\
\hline \multirow[t]{5}{*}{ R3 } & RWW & 20.4 & 39.5 & 34.7 & 40.0 & 33.3 & 50.0 & 25.0 \\
\hline & TWW & 17.9 & 46.7 & 46.7 & 75.0 & 22.2 & 41.7 & 62.5 \\
\hline & WS & 6.9 & 22.7 & 16.0 & 100.0 & 36.4 & 33.3 & 23.5 \\
\hline & Total & 16.2 & 37.9 & 33.7 & 60.0 & 29.6 & 41.7 & 33.3 \\
\hline & & 26.2 & & & 33.3 & & 37.7 & \\
\hline \multirow[t]{5}{*}{ R5 } & RWW & 9.3 & 11.6 & 24.5 & 20.0 & 24.2 & 33.3 & 12.5 \\
\hline & TWW & 14.3 & 26.7 & 36.7 & 25.0 & 22.2 & 16.7 & 25.0 \\
\hline & WS & 6.9 & 18.2 & 12.0 & - & 18.2 & 16.7 & 23.5 \\
\hline & Total & 9.9 & 17.9 & 25.0 & 20.0 & 22.5 & 22.2 & 21.2 \\
\hline & & 13.6 & & & 22.2 & & 21.7 & \\
\hline \multirow{5}{*}{ Class 1 integrons ${ }^{\mathrm{a}}$ (CS3/CS5) } & RWW & $13.0(3.7)$ & $16.3(7.0)$ & $24.5(4.1)$ & - & $27.3(18.2)$ & $25.0(25.0)$ & 12.5 \\
\hline & TWW & 7.1 & $23.3(13.3)$ & $33.3(10.0)$ & 25.0 & $18.5(18.5)$ & $16.7(8.3)$ & $50.0(50.0)$ \\
\hline & WS & 3.4 & $18.2(4.5)$ & $24.0(4.0)$ & 100.0 & 9.1 & $25.0(8.3)$ & 23.5 \\
\hline & Total & $9.0(1.8)$ & $18.9(8.4)$ & $26.9(5.8)$ & 20.0 & $21.1(15.5)$ & $22.2(13.9)$ & $27.3(12.1)$ \\
\hline & & $13.6(4.9)$ & & & $21.0(13.6)$ & & $24.6(13.0)$ & \\
\hline \multirow[t]{5}{*}{ Class 2 integrons } & RWW & 5.6 & 4.7 & 4.1 & - & 6.1 & - & 12.5 \\
\hline & TWW & - & 6.7 & 10.0 & - & 3.7 & 8.3 & 12.5 \\
\hline & WS & - & 9.1 & - & - & - & - & 17.6 \\
\hline & Total & 1.8 & 6.3 & 4.8 & - & 4.2 & 2.8 & 15.2 \\
\hline & & 4.4 & & & 3.7 & & 8.7 & \\
\hline
\end{tabular}

a Class 1 integrons prevalence was estimated on basis of the presence of the integrase gene. Prevalence values corresponding to detection on basis of the regions CS3-CS5 are given between brackets.

leucine mutation in position 83 (due to the transition TCG $\rightarrow$ TTG). Only one (of the 23) WS isolate of the group $\mathrm{B} 22$ did not present this mutation. The aspartate residue in the position 87 of the DNA gyrase was substituted by asparagine (GAC $\rightarrow A A C)$ in 20 isolates and by tyrosine (GAC $\rightarrow$ TAC) in one TWW isolate of group B1. Two isolates, one (of WS) of the type $A_{1}$ and one (of RWW) of group B1 did not present any mutation in position 87 . The serine residue in position 80 of topoisomerase IV was substituted by isoleucine in 17 out of the 23 isolates, in 14 due to the transition AGC $\rightarrow$ ATC and in three due to AGC $\rightarrow$ ATT. Arginine was observed in the position 80 of the topoisomerase IV of four isolates due to the transition AGC $\rightarrow$ AGA. In position 84 , a glutamate residue was substituted by a glycine in an isolate of the type $D_{1}$ from TWW and by a histidine in a $B 2_{2}$ RWW isolate. The genes qnrA, qnrB or qnrS were not detected in any of these isolates. The gene qnrS1 was detected in a single isolate from RWW of the type $A_{1}$, which exhibited an intermediary resistance phenotype against ciprofloxacin.

\section{Discussion}

\subsection{Predominant phylogenetic groups in waste- and surface waters}

In the current study, most of the isolates from wastewater and surface waters could be affiliated to the Clermont et al. (2000) groups A and B1, representing about $60 \%$ of the total in any of the three types of water examined (WS, RWW, TWW). These results are in agreement with previous studies which demonstrated the prevalence of these two groups in environments where the human impact is predominant as in municipal wastewater or in surface water subjected to human-derived contamination (Higgins et al., 2007; Orsi et al. 2007; Sabaté et al., 2008; Mataseje et al., 2009; Radhouani et al., 2009). The least prevalent groups differed in wastewater and in water streams, with the group D being more abundant than B2 in water streams and the opposite being observed in wastewater. Although this observation in the current study could be attributed to the use of distinct culture media for bacteria isolation, similar results were reported by Sabaté et al. (2008), who, in spite of the reduced number of strains studied, detected also higher abundance of $E$. coli of group B2 than of group D in human wastewaters. Moreover, other authors have demonstrated the abundance of isolates of the group D in freshwaters (Hamelin et al., 2007; Higgins et al., 2007).
4.2. Prevalence of antimicrobial resistance and of integrons in the different phylogenetic groups

Groups A and B1 can be considered sister groups, branching together on basis MLST analyses (Gordon et al., 2008). The demonstration that these two groups prevail in humans and in external human-derived environments suggest their frequent co-occurrence (Duriez et al., 2001; Escobar-Páramo et al., 2004; Higgins et al., 2007; Skurnik et al., 2008; Mataseje et al., 2009). Such observations could lead to the hypothesis that in habitats containing high percentages of isolates of the groups A and B1 of human and/or animal origins, they would display similar levels and patterns of antimicrobial resistance and of integrons. In contrast to these expectations, in the current study, the most dramatic differences were observed between groups A and B1. Whereas group A was characterized by low rates of antimicrobial resistance, group B1 was, in general, the group with the highest resistance prevalence. Isolates of group B1 are described as being particularly abundant in animal populations, gathering privileged conditions for resistance acquisition and, thus, for its dissemination (Escobar-Páramo et al., 2004; Higgins et al., 2007; Ishii et al., 2007; Cortés et al., 2010). The high persistence in the environment of isolates of group B1 (Walk et al., 2007) may also explain the higher resistance rates observed, as such fact may represent additional opportunities for gene acquisition and/or organism selection. We can, thus, conclude that isolates of the phylogenetic group B1, given their abundance and high rates of antimicrobial resistance, deserve a special attention when environmental antimicrobial resistance is under analysis.

Isolates of the group B2, in which are included clonal lineages frequently disseminated in the environment and regarded as relevant vectors of extended spectrum-beta-lactamases (Coque et al., 2008; Simões et al., 2010), presented significantly higher rates of resistance against beta-lactams than the isolates of group A. However, in this respect, did not differ significantly from the isolates of the group B1. Further studies will elucidate if the previously referred clonal lineages can be disseminated by waste- and surface waters.

Among the antimicrobial compounds examined in this study it was possible to define a core of resistance phenotypes, observed in high percentages, and often occurring together in the same organism. These antimicrobials comprised the beta-lactams, sulfamethoxazole, tetracycline and streptomycin, all old generation antimicrobial agents. 
Newer and less used drugs, at least at the community level, as ceftazidime, meropenem or colistin were active against almost all isolates (a single exception observed for colistin). The prevalence of R3, R5 and of class 1 integrons followed a similar trend, supporting the hypothesis that the presence class 1 integrons may constitute an advantage for antimicrobial resistance spreading in the waste- and surface waters (Rosser and Young, 1999; Tennstedt et al., 2003; Henriques et al., 2006).

\subsection{Comparison of the genotypes comprising each phylogenetic group}

Recently, Gordon et al. (2008) demonstrated the limitation the PCR-based screening of Clermont et al. (2000) has to identify members of the phylogenetic groups A, B1, B2 and D. Although those authors found some incoherencies when the French or the German multi-locus sequence typing protocol was used, they concluded that each of the two genotypes corresponding to groups $\mathrm{A}, \mathrm{B} 2$ and $\mathrm{D}$, may comprise, in fact, members of distinct phylogenetic groups (Gordon et al., 2008). Such a conclusion led us to hypothesize that in each phylogenetic group, as defined by Clermont et al. (2000), we could observe subgroups yielding distinctive patterns of antimicrobial resistance. To test such hypothesis, we compared the different genotypes comprising each phylogenetic group. This analysis showed the rarity of the type $B 2_{1}$ also referred by Gordon et al. (2008) and the prevalence of the type $A_{0}$. Isolates of the type $A_{0}$ reached almost $25 \%$ of the total, in contrast to the revision made by Gordon et al. (2008), which refers to the low frequency of this type $(<9 \%)$. Even though there are no previous studies describing the ratio $A_{0} / A_{1}$ in waste- and surface waters, we believe that the high prevalence value observed in the current study is characteristic of these types of water.

Isolates of the type $A_{0}$ suffered a significant reduction after wastewater treatment, suggesting that these organisms have lower survival rates during wastewater treatment, than members of the other groups. Moreover, it was observed that isolates of the type $A_{0}$ had significantly lower resistance rates and, surprisingly, among the 111 isolates detected, none was resistant to ciprofloxacin. These observations suggest that isolates of the type $A_{0}$ have a minor role in antimicrobial resistance dissemination in the environment, when compared with those of the type $A_{1}$. Antimicrobial resistance prevalence among the isolates of the type $A_{1}$ was intermediate between that observed for group B1 and for isolates of the type $A_{0}$. Such an intermediary value may hint the poor resolution of the phylogenetic groups A and B1, when the Clermont et al. (2000) method is used. In fact, it has been referred to that some organisms of the phylogenetic group B1 may be misclassified as $A_{1}$ (Gordon et al., 2008). Similarly, it has been evidenced that besides $E$. coli of group $A$ others, belonging to other phylogenetic groups, may yield the type $A_{0}$ when tested with the Clermont et al. (2000) protocol (Gordon et al., 2008). Although we cannot exclude the suspect that some non-group A E. coli evidenced this genotype in the current study, we observed a coherent and highly distinctive antimicrobial resistance patterns in these organisms.

An intriguing result was the apparent increase in the prevalence of ciprofloxacin and streptomycin resistance among the TWW isolates, when compared with those from the raw effluent. A detailed analysis of the genotypes comprising each phylogenetic group hints a possible explanation for these results. According to our data, the dynamic of the different population subsets during wastewater treatment explain these significant increases of ciprofloxacin and streptomycin resistance. The proportion of isolates of the type $A_{0}$ in which no ciprofloxacin resistance was observed, suffered a significant reduction after wastewater treatment, suggesting that, in fact, it was the percentage of isolates with no ciprofloxacin resistance that decreased, rather than a possible selection of ciprofloxacin resistant organisms or even the spreading through horizontal gene transfer. This is consistent with the gene mutations observed to explain the ciprofloxacin resistance and the failure to detect the plasmid associated qnr genes in the same isolates. The elimination during wastewater treatment of isolates of the type $A_{0}$ seems the most plausible explanation for the observed increase on ciprofloxacin resistance. In the same way, the rearrangement of the population subsets after wastewater treatment could explain the apparent increase of resistance to streptomycin among the TWW isolates. As observed for ciprofloxacin, $\mathrm{A}_{0}$ isolates had significantly lower resistance prevalence to streptomycin, and thus the same effect was observed for this antibiotic. In this case, also the variations on the percentages of isolates of the group D contributed for the observed effect. Streptomycin resistance was significantly more prevalent among the isolates of group D than in those B1 or A and, simultaneously, isolates of group D were more prevalent in the TWW than in the RWW. A similar rearrangement of bacterial groups during wastewater treatment was reported before for enterococci (Ferreira da Silva et al., 2006). In that study, with municipal wastewater isolates, members of the species Enterococcus hirae, with very low levels of antimicrobial resistance, were eliminated more extensively during wastewater treatment than members of the species Enterococcus faecium and Enterococcus faecalis, originating, as in the present study, an apparent increase in ciprofloxacin resistance in the treated effluent (Ferreira da Silva et al., 2006).

\section{Conclusions}

This study demonstrates that $E$. coli of the phylogenetic group B1 have a major role in antimicrobial resistance dissemination in the environment. Significant increases of ciprofloxacin and streptomycin resistance prevalence after wastewater treatment were observed to result from rearrangements of different population subsets. Different E. coli subsets, namely of the type $A_{0}$, which were preferentially eliminated during wastewater treatment contributed to the observed increases of antimicrobial resistance prevalence in the final effluent. These findings show that horizontal gene transfer and the selection of resistant organism/genetic determinant may not represent the only mechanisms contributing for antimicrobial resistance increase in the environment - the preferential elimination of susceptible organisms rather than their resistant counterparts may have an important contribute for resistance increase.

\section{Acknowledgements}

The authors gratefully acknowledge Professor Patrice Nordmann for the controls for qnr genes detection. This study was financed by the Fundação para a Ciência e a Tecnologia (project PTDC/AMB/71236/ 2006).

\section{References}

Cattoir V, Poirel L, Rotimi V, Soussy C-J, Nordmann P. Multiplex PCR for detection of plasmid-mediated quinolone resistance qnr genes in ESBL-producing enterobacterial isolates. J Antimicrob Chemother 2007;60:394-7.

Clermont O, Bonacorsi S, Bingen E. Rapid and simple determination of the Escherichia coli phylogenetic group. Appl Environ Microbiol 2000;66:4555-8.

Clinical and Laboratory Standards Institute. Performance standards for antimicrobial susceptibility testing: M100-S17, Vol. $27 \mathrm{Nr}$. 1. PA: Wayne; 2007.

Coque TM, Novais A, Carattoli A, Poirel L, Pitout J, Peixe L, et al. Dissemination of clonally related Escherichia coli strains expressing extended-spectrum beta-lactamase CTX-M-15. Emerg Infect Dis 2008;14:195-200.

Cortés P. Blanc V, Mora A, Dahbi G, Blanco JE, Blanco M, et al. Isolation and characterization of potentially pathogenic antimicrobial-resistant Escherichia col strains from chicken and pig farms in Spain. Appl Environ Microbiol 2010;76: 2799-805.

Duriez P, Clermont O, Bonacorsi S, Bingen E, Chaventré A, Elion J, et al. Comensal Escherichia coli isolates are phylogenetically distributed among geographically distinct human populations. Microbiology 2001;174:1671-6.

Escobar-Páramo P, Grenet K, Le Menac'h A, Rode L, Salgado E, Amorin C, et al. Large-scale population structure of human commensal Escherichia coli isolates. Appl Environ Microbiol 2004; 70:5698-700. 
Ferreira da Silva M, Tiago I, Veríssimo A, Boaventura AR, Nunes OC, Manaia CM. Antibiotic resistance of enterococci and related bacteria in an urban wastewater treatment plant. FEMS Microbiol Ecol 2006;55:322-9.

Ferreira da Silva M, Vaz-Moreira I, Gonzalez-Pajuelo M, Nunes OC, Manaia CM. Antimicrobial resistance patterns in Enterobacteriaceae isolated from an urban wastewater treatment plant. FEMS Microbiol Ecol 2007:60:166-76.

Garcia-Aljaro C, Moreno E, Andreu A, Prats G, Blanch AR. Phylogroups, virulence determinants and antimicrobial resistance in $s x_{2}$ gene-carrying Escherichia coli isolated from aquatic environments. Res Microbiol 2009;160:585-91.

Gordon DM, Clermont O, Tolley H, Denamur E. Assigning Escherichia coli strains to phylogenetic groups: multi-locus sequence typing versus the PCR triplex method. Environ Microbiol 2008;10:2484-96.

Hamelin K, Bruant G, El-Shaarawi A, Hill S, Edge TA, Fairbrother J, et al. Occurrence of virulence and antimicrobial resistance genes in Escherichia coli isolates from different aquatic ecosystems within the St. Clair river and Detroit river areas. Appl Environ Microbiol 2007;73:477-84.

Henriques IS, Fonseca F, Alves A, Saavedra MJ, Correia A. Occurrence and diversity of integrons and $\beta$-lactamase genes among ampicillin-resistant isolates from estuarine waters. Res Microbiol 2006;157:938-47.

Herzer PJ, Inouye S, Inouye M, Whittam TS. Phylogenetic distribution of branched RNAlinked multicopy single-stranded DNA among natural isolates of Escherichia coli. J Bacteriol 1990;172:6175-81.

Higgins J, Hohn C, Hornor S, Frana M, Denver M, Joerger R. Genotyping of Escherichia coli from environmental and animal samples. J Microbiol Meth 2007;70:227-35.

Ishii S, Meyer KP, Sadowsky MJ. Relationship between phylogenetic groups, genotypic clusters, and virulence gene profiles of Escherichia coli strains from diverse human and animal sources. Appl Environ Microbiol 2007;73:5703-10.

International Organization for Standardization (ISO) 9308-1:2000. Water quality detection and enumeration of Escherichia coli and coliform bacteria - Part 1: membrane filtration method. Geneva: 2000 International Organization for Standardization; 2000. ISO 9308-1:2000.

Laroche E, Pawlak B, Berthe T, Skurnik D, Petit F. Occurrence of antibiotic resistance and class 1, 2 and 3 integrons in Escherichia coli isolated from a densely populated estuary (Seine, France). FEMS Microbiol Ecol 2009;11:1-13.

Lévesque C, Piché L, Larose C, Roy PH. PCR mapping of integrons reveals several novel combinations of resistance genes. Antimicrob Agents Chemother 1995;39:185-91.

Mataseje LF, Neumann N, Crago B, Baudry P, Zhanel GG, Louie M, et al. Characterization of cefoxitin-resistant Escherichia coli isolates from recreational beaches and private drinking water in Canada between 2004 and 2006. Antimicrob Agents Chemother 2009;53:3126-30

Maynard C, Bekal S, Sanschagrin F, Levesque RC, Brousseau R, Masson L, et al. Heterogeneity among virulence and antimicrobial resistance gene profiles of extraintestinal Escherichia coli isolates of animal and human origin. J Clin Microbiol 2004:42:5444-52

McDonald LC, Chen FJ, Lo HJ, Yin HC, Lu PL, Huang CH, et al. Emergence of reduced susceptibility and resistance to fluoroquinolones in Escherichia coli in Taiwan and contributions of distinct selective pressures. Antimicrob Agents Chemother 2001;45: 3084-91.

Orsi RH, Stoppe NC, Sato MI, Gomes TA, Prado PI, Manfio GP, et al. Genetic variability and pathogenicity potential of Escherichia coli isolated from recreational water reservoirs. Res Microbiol 2007;158:420-7.

Radhouani H, Poeta P, Igrejas G, Gonçalves A, Vinué L, Torres C. Antimicrobial resistance and phylogenetic groups in isolates of Escherichia coli from seagulls at the Berlengas nature reserve. Vet Rec 2009;165:138-42.

Rijavec M, Erjavec MS, Avgustin JA, Reissbrodt R, Fruth A, Krizan-Hergouth V, et al. High prevalence of multidrug resistance and random distribution of mobile genetic elements among uropathogenic Escherichia coli (UPEC) of the four major phylogenetic groups. Curr Microbiol 2006;53:158-62.

Rosser SJ, Young HK. Identification and characterization of class 1 integrons in bacteria from an aquatic environment. J Antimicrob Chemother 1999;44:11-8.

Sabaté M, Prats G, Moreno E, Ballesté E, Blanch AR, Andreu A. Virulence and antimicrobial resistance profiles among Escherichia coli strains isolated from human and animal wastewater. Res Microbiol 2008;159:288-93.

Simões RR, Poirel L, Da Costa PM, Nordmann P. Seagulls and beaches as reservoirs for multidrug-resistant Escherichia coli. Emerg Infect Dis 2010;16:110-2.

Skurnik D, Le Menac'h A, Zurakowski D, Mazel D, Courvalin P, Denamur E, et al. Integron-associated antibiotic resistance and phylogenetic grouping of Escherichia coli isolates from healthy subjects free of recent antibiotic exposure. Antimicrob Agents Chemother 2005;49:3062-5.

Skurnik D, Bonnet D, Bernede-Bauduin C, Michel R, Guette C, Becker JM, et al. Characteristics of human intestinal Escherichia coli with changing environments. Environ Microbiol 2008;10:2132-7.

Tennstedt T, Szczepanowski R, Braun S, Pühler A, Schlüter A. Occurrence of integronassociated resistance gene cassettes located on antibiotic resistance plasmids isolated from a wastewater treatment plant. FEMS Microbiol Ecol 2003;45:239-52.

Walk ST, Alm EW, Calhoun LM, Mladonicky JM, Whittam TS. Genetic diversity and population structure of Escherichia coli isolated from freshwater beaches. Environ Microbiol 2007;9:2274-88.

Weigel LM, Steward CD, Tenover FC. gyrA mutations associated with fluoroquinolone resistance in eight species of Enterobacteriaceae. Antimicrob Agents Chemothe 1998;42:2661-7. 